\title{
A brief discussion on the relationship between apparent stress and slip-weakening law based on the energy partition criteria*
}

\author{
Boyan Liu ${ }^{\star}$ and Baoping Shi \\ College of Earth Science, Graduate University of Chinese Academy of Sciences, Beijing 100049, China
}

\begin{abstract}
According to the representation theorem of seismic energy radiation, we know that, at any point on the fault, the instantaneous seismic radiation energy, $E_{\mathrm{S}}$, the seismic moment $M_{0}$, and the apparent stress $\sigma_{\mathrm{a}}=\mu E_{\mathrm{S}} / M_{0}(\mu$ is the shear modulus on the fault plane) should be positive values at any time during an earthquake faulting. However, we have noticed that, in recent source parameter inversion scheme for deriving the critical slip-weakening distance, the apparent stress used as a constraint condition on the fault plane could be less than zero or negative value, and the negative part was considered as dissipation energy and incorporated to the fracture energy. Although the mathematical formula in such case has no influence to the final resolution, however, the earthquake dynamic source process violates obviously the basic physical law, which could results in the overestimating of radiated seismic energy. In this study, we have proposed an alternative way to take account of the apparent stress expression based on the earthquake energy partition principle, and at the same time, we have also suggested that an additional constraint regarding to the radiation energy conservation on the fault could be added into the source parameter inversion in order to estimate the critical slip-weakening distance $D_{\mathrm{c}}$.
\end{abstract}

Key words: apparent stress; seismic radiation energy; fracture energy; critical slip-weakening distance CLC number: P315.3 Document code: A

\section{Introduction}

From our current understanding of earthquake source mechanism, we know that, as fault ruptures with associated slip along the fault plane, the radiated seismic energy, $E_{\mathrm{S}}$, from the fault should not be less than zero, so does the seismic moment, $M_{0}$. Correspondingly, apparent stress, $\sigma_{\mathrm{a}},\left(\sigma_{\mathrm{a}}=\mu E_{\mathrm{S}} / M_{0}, \mu\right.$ is a material shear module on the fault) should also be positive. Apparent stress $\sigma_{\mathrm{a}},\left[\sigma_{\mathrm{a}}=E_{\mathrm{S}} /\left(\Sigma_{0} D^{f}\right)\right.$, where $\Sigma_{0}$ and $D^{f}$ are the fault ruptured area and averaged slip displacement, respectively] describes the amount of radiation energy per unit area and per unit slip. Therefore, the apparent stress $\sigma_{\mathrm{a}}$ could not be less than zero at any time during fault rupture process.

In recent study given by Pulido and Irikura (2000), apparent stress, $\sigma_{\mathrm{a}}$, has been used as a constraint to resolve the critical slip-weakening distance, $D_{\mathrm{c}}$, for the $1992 M_{\mathrm{W}} 7.3$ Landers earthquake, California. In their

\footnotetext{
* Received 3 March 2008; accepted in revised form 23 October 2008; published 10 February 2009.

• Corresponding author. e-mail: liuboyan06@mails.gucas.ac.cn
}

inversion algorithm, they mentioned that the apparent stress, $\sigma_{\mathrm{a}}$, could be a negative at some times (Madariaga, 1977), and the negative part does not contribute to the radiated seismic energy, but belongs to an energy dissipation which could be combined to the fracture energy used to advance the rupture propagation. A generalized mathematical description is as follows (Madariaga, 1977; Pulido and Irikura, 2000)

$$
\sigma_{\mathrm{a}}(D)=\frac{1}{D \Sigma_{0}} \int_{\Sigma_{0}} \mathrm{~d} S \int_{0}^{D} \mathrm{~d} u\left[\bar{\sigma}_{i j}-\sigma_{i j}(u)\right] n_{j}-\frac{\gamma_{\text {eff }}}{D},
$$

where $\bar{\sigma}_{i j}=\left(\sigma_{i j}^{0}+\sigma_{i j}^{1}\right) / 2$ is an average shear stress on fault, and $\sigma_{i j}^{0}$ and $\sigma_{i j}^{1}$ are the shear stresses before and after earthquake, respectively; $D$ is an accumulation slip displacement on fault surface of $\Sigma_{0}$, and $\sigma_{i j}(u)$ is a time-dependent/slip-dependent frictional stress on fault. $n_{j}$ is a unit vector which is perpendicular to fault surface of $\Sigma_{0}, \gamma_{\text {eff }}$ is the effective surface energy on $\Sigma_{0}$. In general, because the effective surface energy ' $\gamma_{\text {eff }}$ ' is very difficult to estimate, it can usually be treated as part of fracture energy (Rivera and Kanamori, 2005). Combined with 
simplified slip-weakening frictional law indicated in Figure 1, the apparent stress, $\sigma_{\mathrm{a}}$, derived from equation (1) increases with slip (see Figure 1) gradually. In Figure 1 , when $D \leq D^{\prime}$, then $\sigma_{\mathrm{a}} \leq 0$, the area given by triangle $A O D^{\prime}$ corresponds to a dissipation energy; while $D \geq D^{\prime}$, then $\sigma_{\mathrm{a}} \geq 0$, it corresponds to radiated seismic energy. Once $D$ is equal to $D_{\mathrm{c}}\left(D_{\mathrm{c}}\right.$ is called a critical slip-weakening distance), $\sigma_{\mathrm{a}}$ reaches the maximum value. The resultant total radiated seismic energy is given by the area of trapeziform $B D^{\prime} D^{f} C$. According to earthquake energy partition principle (Rivera, Kanamori, 2005), the actual radiated seismic energy should be equal to the area given by a rectangle $B D_{c} D^{f} C\left(E_{\mathrm{S}}=\left(\sigma_{0}-\sigma_{1}\right)\left(D^{f}-D_{\mathrm{c}}\right) \Sigma_{0} / 2\right)$. In this case, the radiated seismic energy calculated by equation (1) does not match with the energy partition principle for a source parameter inversion scheme in an implementation level.

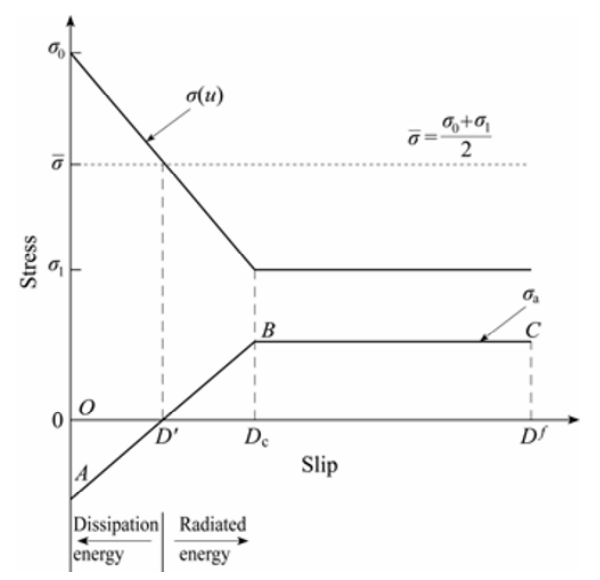

Figure 1 The changes of apparent stress and average stress in the slipping. $D_{\mathrm{c}}$ is the critical slip weakening distance, $\sigma_{0}$ and $\sigma_{1}$ are the initial stress and final stress on the fault, $\bar{\sigma}$ is the average stress of $\sigma_{0}$ and $\sigma_{1}$. The upper and lower lines are frictional stress and apparent stress, respectively. Horizontal axis represents fault slip.

Obviously, the negative apparent stress appeared in Figure 1 is conflicted with earthquake energy partition principle. In this study, by using earthquake energy partition principle, we try to point out the shortage of equation (1) used in source parameter inversion without any modification. We also give an alternative and correct description of apparent stress, $\sigma_{\mathrm{a}}$, in order to derive source parameters correctly without violating earthquake energy partition principle.

\section{Energy partition principle}

Theoretically, the fault is covered in a closed sur- face $S_{0}$ (outer boundary), with a characteristic scale $\Sigma$ (internal boundary) $\left(\sum=\Sigma^{-}+\Sigma^{+}\right)$. When a relative motion of both fault sides occurs, some part of energy radiates to $S_{0}$ in the form of seismic waves. If the distance $r$ from any point $\xi$ on the fault to $S_{0}$ is much larger than fault size, the radiation energy from far field can be written as follows (Rudnicki and Freund, 1981):

$$
E_{\mathrm{S}}=\int_{t_{0}}^{t_{1}} \int_{S_{0}} \rho\left[\alpha\left(\dot{u}_{j} n_{j}\right)^{2}+\beta\left(\dot{u}_{i}-\dot{u}_{j} n_{j} n_{i}\right)^{2}\right] \mathrm{d} S \mathrm{~d} t,
$$

where $\rho$ is density of homogeneous isotropic medium, $\alpha$ and $\beta$ are $\mathrm{P}$-wave and $\mathrm{S}$-wave speed, respectively, $u_{j}$ is the displacement on the boundary of $S_{0}, n_{j}$ is its unit normal vector, $t_{0}$ is time before earthquake, and $t_{1}$ is stop time of particle motion on $S_{0}$ after earthquakes.

On the other side, if the distance $\boldsymbol{r}$ from any point $\xi$ on the fault to $S_{0}$ is much larger than fault size, the seismic radiation energy (only considering S-wave) led from the change of elastic potential energy before and after earthquakes can be calculated (Kostrov, 1974; Dahlen, 1977):

$$
\begin{aligned}
E_{\mathrm{S}}= & \frac{1}{2} \int_{\Sigma_{0}}\left(\sigma_{i j}^{0}+\sigma_{i j}^{1}\right) \Delta u_{i}^{1} v_{j} \mathrm{~d} S-\int_{\Sigma} 2 \gamma_{\text {eff }} \mathrm{d} S \\
& -\int_{\Sigma} \mathrm{d} S \int_{t_{r}(\xi)}^{t_{1}} \sigma_{i j} \Delta \dot{u}_{i} v_{j} \mathrm{~d} t,
\end{aligned}
$$

where $\sigma_{i j}^{0}$ and $\sigma_{i j}^{1}$ are the shear stresses before and after earthquake, respectively. $\sigma_{i j}$ is the stress on the fault at any time during fault rupture. $\Delta u_{i}=u_{i}^{+}-u_{i}^{-}$is a relative slip displacement of the two sides of the fault. $\Delta u_{i}^{1}$ is the final slip displacement. $v_{j}$ is a unit vector perpendicular to the fault $\Sigma_{0} \cdot t_{r}(\xi)$ is the time on which the slip begins from any point $\xi$ on the fault. $\gamma_{\text {eff }}$ is effective surface energy on $\Sigma_{0}$ (Kostrov, 1974). Combining with equations (2) and (3), the observation seismic signals gathered on the ground surface can be used to estimate the source dynamic rupture parameters on the fault (energy of P-wave radiated from double couple source is much less than the energy of S-wave). More detail discussions can be founded in Rivera and Kanamori (2005). Furthermore, equation (3) can also be written to (Rivera and Kanamori, 2005):

$$
\begin{aligned}
E_{\mathrm{S}}= & \frac{1}{2} \int_{\Sigma_{0}}\left(\sigma_{i j}^{0}+\sigma_{i j}^{1}\right) \Delta u_{i}^{1} v_{j} \mathrm{~d} S-\int_{\Sigma_{0}} 2 \gamma_{\text {eff }} \mathrm{d} S \\
& -\int_{\Sigma_{0}} \mathrm{~d} S \int_{0}^{\Delta u_{i}^{1}} \sigma_{i j}\left(\Delta u_{i}\right) v_{j} \mathrm{~d}\left(\Delta u_{i}\right) .
\end{aligned}
$$

In fact, we know that, from equation (4), the radiated seismic energy not only depends on slip behavior at 
a single point on fault, but also depends on the slip processes on the whole fault surface, which was expounded by Rivera and Kanamori (2005). Therefore, the theoretical study given by Rivera and Kanamori (2005) indicated that a simple summation of seismic energy flow from an individual point on the fault resulted in an in-corrected estimating of total radiated seismic energy. In this way, we can get this by the first integral of equation (4), which describes the volume integral of any pipe covered with $\mathrm{d} S$, rather than the integral of $\mathrm{d} S$ (see Figure 2, Rivera and Kanamori, 2005).

If the stress change on the fault surface and the particle displacement obey simplified slip-weakening principle (Figure 2), the equation (4) can be rewritten as

$$
\begin{aligned}
E_{\mathrm{S}} & =\frac{1}{2} \int_{\Sigma_{0}}\left(\sigma_{0}+\sigma_{1}\right) \Delta u^{1} \mathrm{~d} S \\
& -\int_{\Sigma_{0}} \mathrm{~d} S \int_{0}^{\Delta u^{1}} \sigma(u) \mathrm{d} u-\int_{\Sigma_{0}} 2 \gamma_{\text {eff }} \mathrm{d} S .
\end{aligned}
$$

In equation (5), if we change the surface integral into the direction of fault surface, the $\sigma_{i j}^{0}$ and $\sigma_{i j}^{1}$ can be replaced by $\sigma_{0}$ and $\sigma_{1}$, in which $\sigma_{0}$ and $\sigma_{1}$ are the initial and final stress on the fault, respectively, $\Delta u^{1}$ is the final slip gap (single point source approach), $\sigma(u)$ is a time-dependent/slip-dependent stress (dynamic frictional stress) on the fault during fault rupture propagation. According to the definition of apparent stress (Wyss and Brune, 1968), we have:

$$
\begin{aligned}
\sigma_{\mathrm{a}} & =\mu \frac{E_{\mathrm{S}}}{M_{0}}=\frac{E_{\mathrm{S}}}{\Delta u^{1} \Sigma_{0}} \\
& =\frac{1}{\Delta u^{1} \Sigma_{0}} \int_{\Sigma} \mathrm{d} S \int_{0}^{\Delta u^{1}} \mathrm{~d} u[\bar{\sigma}-\sigma(u)]-\frac{2}{\Delta u^{1}} \gamma_{\mathrm{eff}},
\end{aligned}
$$

where $\bar{\sigma}=\left(\sigma_{0}+\sigma_{1}\right) / 2$. In general, the surface energy $\gamma_{\text {eff }}$ in equations (5) and (6) can be involved into fracture energy for the specific calculation, which means that the last term in the right side of equations (5) and (6) can be neglected. In contrasting, although the form of equation (6) and equation (1) seems similar with each other, the physical meaning related to the rupture process is the kind of difference. In equation (6), the integral upper limit of slip is final slip $\Delta u^{1}$, but the integral upper limit of slip in equation (1) is an instantaneous accumulation slip $D$ (the slip of each point on the fault). Therefore, by neglecting the surface energy or distributing it to the rupture energy $E_{\mathrm{G}}$ (see Figure 2) (Rivera and Kanamori, $2005)$, the apparent stress, $\sigma_{\mathrm{a}}$, given by equation (6) is always greater than or equal to zero.



Figure 2 Simplified slip-weakening law. $D_{c}$ is the critical slip weakening distance, $\sigma_{0}$ and $\sigma_{1}$ are initial stress and final stress in the fault, respectively. $\bar{\sigma}$ is the average stress of $\sigma_{0}$ and $\sigma_{1}, \sigma_{r}$ is the average stress of $\sigma(u)$ and $\sigma_{f}$ in the total rupture and slip process. According the energy distribution principle, the area of $\triangle A C D, \triangle A B C$ and rectangle $B E F D$ are seismic radiation energy $E_{\mathrm{S}}$, fracture energy $E_{\mathrm{G}}$, and heat, respectively.

With a comparison between equations (1) and (2), we know that the apparent stress, $\sigma_{\mathrm{a}}$, in equation (1) perhaps negative in some times comes from an ambiguous description of the average stress $\bar{\sigma}$. In equation (1), the averaged stress is given by $\bar{\sigma}=\left(\sigma_{0}+\sigma_{1}\right) / 2$ in which $\sigma_{1}$ is a dynamic fractional stress and equals to the stress corresponding to final $\operatorname{slip} \Delta u^{1}$, but the integral upper limit $D$ given in equation (1) is an instantaneous accumulation slip. As a result, when $D \leq D^{\prime}$, $\int_{0}^{D} \sigma(u) \mathrm{d} u \geq \int_{0}^{D} \bar{\sigma}\left(\Delta u^{1}\right) \mathrm{d} u$, resultant to $\sigma_{\mathrm{a}} \leq 0$. In order to solve the negative apparent stress $\sigma_{\mathrm{a}}$ appeared in equation (1), we change the integral upper limit of time $t_{1}$ to instantaneous time $t\left(0 \leq t \leq t_{1}\right)$ according to equation (3). In this case, corresponding to time $t$, the integral upper limit of slip in equation (5) changes to $D$, and correspondingly, $\Delta u^{1}=D, \quad \sigma_{1}=\sigma(D), \quad \bar{\sigma}=\left[\sigma_{0}+\sigma(D)\right] / 2$, respectively. After such modification, $\bar{\sigma}=\left[\sigma_{0}+\sigma(D)\right] / 2$ becomes an instantaneous averaged stress, which depends on the time or slip process. The radiated seismic energy can be calculated by:

$$
\begin{aligned}
E_{\mathrm{S}}(D)= & \int_{\Sigma_{0}} \mathrm{~d} S \int_{0}^{D} \frac{1}{2}\left[\sigma_{0}+\sigma(D)\right] \mathrm{d} u \\
& -\int_{\Sigma_{0}} \mathrm{~d} S \int_{0}^{D} \sigma(u) \mathrm{d} u
\end{aligned}
$$

From equation (7), we can see that, when $D \leq D_{\mathrm{c}}$, we always have $\sigma(D) \geq \sigma_{1}$ and $\bar{\sigma}=\left[\sigma_{0}+\sigma(D)\right] / 2 \geq \sigma(D)$. 
The integral of the first and second terms in the right side of equation (7) are shown in Figure 3. From Figure 3 , we can see that, when $D \leq D_{c}, E_{\mathrm{S}}=0$, while $D \geq D_{\text {c }}$, $E_{\mathrm{S}}(D)$ increases linearly with $\operatorname{slip} D$. When $\sigma(D)=\sigma_{1}$, the averaged stress of $\bar{\sigma}=\left[\sigma_{0}+\sigma_{1}\right] / 2$ becomes a constant. In this way, $\sigma_{\mathrm{a}}$ is always a constant. Therefore, equation (1) should be rewritten as

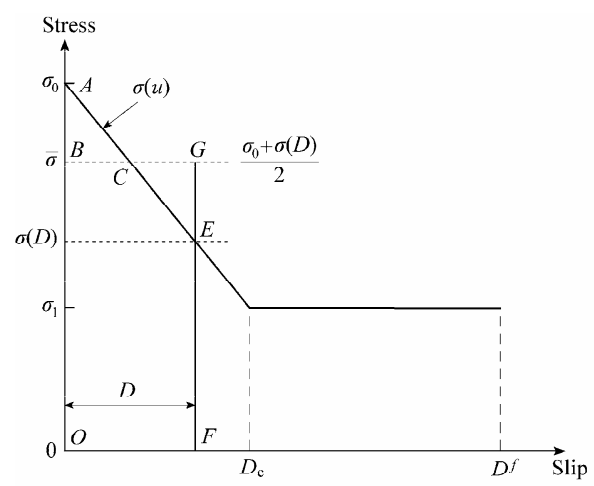

(a)

$$
\sigma_{\mathrm{a}}(D)=\frac{1}{D \Sigma_{0}} \int_{\Sigma_{0}} \mathrm{~d} S \int_{0}^{D}\left\{\frac{1}{2}\left[\sigma_{0}+\sigma(D)\right]-\sigma(u)\right\} \mathrm{d} u .
$$

Figure 4 shows that the apparent stress in equation (8) increases with slip. Compared with Figure 1, the apparent stress $\sigma_{\mathrm{a}}$ in equation (8) is always greater than or equal to zero, and reaches to the maximum value in

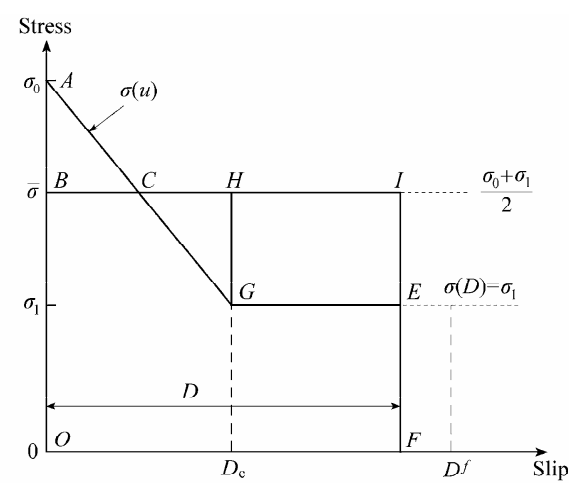

(b)

Figure 3 Representation of equation (7) when $D<D_{c}$ (a) and $D>D_{\mathrm{c}}$ (b). When $D<D_{\mathrm{c}}$, the first term is the area of rectangle $B O F G$, while the second term is the area of trapezoid $A O F E$. Due to the area of $\triangle A B C$ equals to the area of $\triangle C E G$, the area of $B O F G$ and area of $A O F E$ are the same. In this case, $E_{\mathrm{S}}$ is always zero all the time; When $D$ $>D_{\mathrm{c}}$, the first term is the area of rectangle BOFI too, while the second term is the area of AOFEG. Because the area of $\triangle A B C$ is equal to the area of $\triangle C G H$, the area of $B O F I$ is always larger than the area of $A O F E G$; resultant a net difference of area of $H G E I$. In this case, $E_{\mathrm{S}}$ is just the area of $H G E I$.

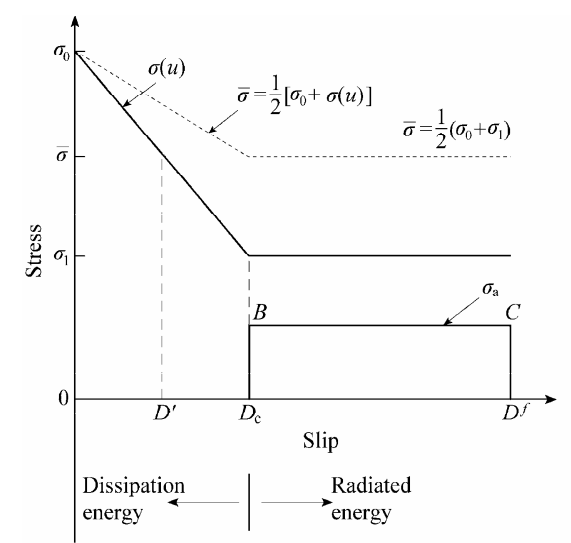

(a)

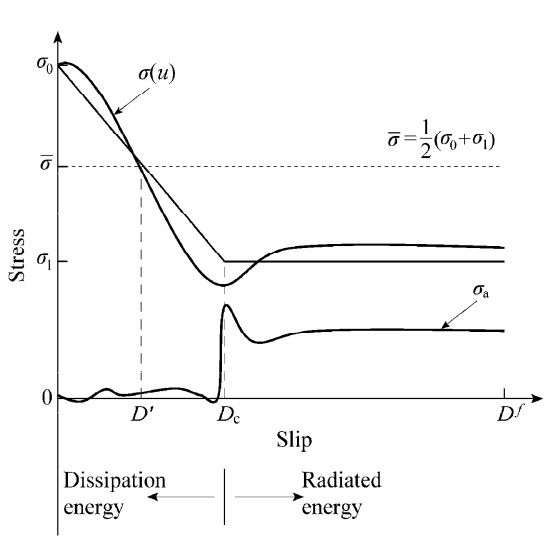

(b)

Figure 4 Time-dependent/Slip-dependent variations of apparent stress of $\sigma_{\mathrm{a}}$ with slip increase under a slip-weakening law. (a) A simplified slip-weakening model. When $D<D_{\mathrm{c}}, \sigma_{\mathrm{a}}=0$, and when $D \geq D_{\mathrm{c}}$, The seismic energy is radiated from fault plane associated with fault rupture motion. In this case, $\sigma_{\mathrm{a}}$ becomes a constant on the fault. The instantaneous stresses are shown as by the dashed line; (b) A general slip-weakening model $\sigma(u)$ and the variation of $\sigma_{\mathrm{a}}$ showed as the solid line.

$D=D_{\mathrm{c}}$. It is remarkable that, the radiated seismic energy from equation (8) is always conserved with $E_{\mathrm{S}}=\left(\Delta u^{1}-D_{\mathrm{c}}\right)$ $\left(\sigma_{0}-\sigma_{1}\right) \Sigma_{0} / 2$. From this study, we suggest that, in earthquake source parameter inversion, we should not only use apparent stress $\sigma_{\mathrm{a}}$ as a constraint, but also need to take the radiated seismic energy $E_{\mathrm{S}}$ as an additional con- straint condition in order to improve the derived precision of $D_{\mathrm{c}}$, the critical slip-weakening distance.

\section{Conclusions}

With the slip-weakening model, Madariaga (1977) first proposed the variation of apparent stress $\sigma_{\mathrm{a}}$ with 
slip. In recent source parameter inversion, this formula has been used as a constrained condition to derive rupture speed variation and the critical slip distance distribution pattern on the fault for the $1992 M_{\mathrm{W}} 7.3$ Landers earthquake, California (Pulido and Irikura, 2000). In the description of apparent stress $\sigma_{\mathrm{a}}$, Pulido and Irikura (2000) referred to that the apparent stress could be less than zero in which the negative value was considered as the dissipation energy and incorporated to the fraction energy (Madariaga, 1977). According to earthquake energy partition principle, we proposed an alternative way to take account of the apparent stress expression in order to avoid such a non-physical phenomenon. Although, the former description might have no influence to the solution of critical slip-weakening distance $D_{\mathrm{c}}$, but it could often overestimate the radiated seismic energy and artificially add to certain uncertainty in source parameter estimation. Through a consideration of the average stress varying instantaneously with time or slip during fault rupture process, we have derived an alternative description of time-dependent/slip-dependent apparent stress formula based on the slip-weakening model without breaching physical principle. In addition, a new way used to estimate the critical slip-weakening distance
$D_{\mathrm{c}}$ and radiated seismic energy $E_{\mathrm{S}}$ simultaneously is proposed based on our study.

Acknowledgements This study was supported by Foundation for Seismological Researches of the Institute of Crustal Dynamics, China Earthquake Administration, (ZDJ2007-1), One Hundred Individual Program of Chinese Academy of Sciences and National Natural Science Foundation of China (40574022).

\section{References}

Dahlen F A (1977). The balance of energy in earthquake faulting. Geophys $J$ R astr Soc 48: 239-261.

Kostrov B (1974). Seismic moment and energy of earthquakes, and seismic flow of rock. Izv Earth Physics 1: 23-40.

Madariaga R (1977). Implications of stress drop models of earthquakes for the inversion of stress drop from seismic observations. Pure Appl Geophys 115: 301-315.

Pulido N and Irikura K (2000). Estimation of dynamic rupture parameters from the radiated seismic energy and apparent stress. Geophys Res Lett 27: 3 945-3 948.

Rivera L and Kanamori H (2005). Representations of the radiated energy in earthquakes. Geophys J Int 162: 148-155.

Rudnicki J W and Freund L B (1981). On energy radiation from seismic sources. Bull Seism Soc Amer 71: 583-595.

Wyss M and Brune J N (1968). Seismic moment, stress, and source dimensions for earthquakes in the California-Nevada region. $J$ Geophys Res 73: 4 681-4 694. 